\title{
Stage IIA1
}

National Cancer Institute

\section{Source}

National Cancer Institute. Stage IIA1. NCI Thesaurus. Code C95177.

A stage term that applies to cervical cancer and indicates that the tumor invades beyond the uterus but not to the pelvic wall or to the lower third of vagina. There is no parametrial invasion. The lesion is clinically visible and measures $4.0 \mathrm{~cm}$ or less in greatest dimension. 